\title{
Estimativas da evapotranspiração de referência em diferentes condições de nebulosidade
}

\author{
Adilson Pacheco de Souza(1), Daniel Fonseca de Carvalho(2), Leonardo Batista Duarte da Silva(2), \\ Frederico Terra de Almeida ${ }^{(1)}$ e Hermes Soares da Rocha(2)
}

\begin{abstract}
(1)Universidade Federal de Mato Grosso, Instituto de Ciências Agrárias e Ambientais, Avenida Alexandre Ferronatto, no 1.200, Campus Sinop, CEP 78557-267 Sinop, MT. E-mail: adilsonpacheco@ufmt. br, fredterr@gmail.com (2)Universidade Federal Rural do Rio de Janeiro, Instituto de Tecnologia, Departamento de Engenharia, BR 465, Km 07, CEP 23890-000 Seropédica, RJ. E-mail: carvalho@ufrrj.br, irriga@ufrrj.br, hermessrocha@hotmail.com
\end{abstract}

Resumo - O objetivo deste trabalho foi avaliar o desempenho de métodos de estimativa da evapotranspiração de referência (ETo), em diferentes condições de nebulosidade, no Município de Seropédica, RJ. As estimativas de ETo, entre 1/6/2006 e 31/7/2007, foram comparadas com medidas diárias realizadas em lisímetro de pesagem, com agrupamento total dos dados e com a discretização pela variação do índice de claridade diário $\mathrm{K}_{\mathrm{T}}$ em quatro classes: $\mathrm{K}_{\mathrm{T}} \leq 0,35$, nublado (Nub); $0,35<\mathrm{K}_{\mathrm{T}} \leq 0,55$, céu parcialmente nublado (PNAd); $0,55<\mathrm{K}_{\mathrm{T}} \leq 0,65$, céu parcialmente aberto (PNAb); e $\mathrm{K}_{\mathrm{T}}>0,65$, céu aberto $(\mathrm{Ab})$. Para avaliação da qualidade das estimativas realizadas pelos diferentes métodos, foram empregados o erro absoluto médio (MBE), a raiz quadrada do quadrado médio do erro (RMSE), e índices de ajustamento e de desempenho. No agrupamento total dos dados, os métodos Penman-Monteith FAO (PMF) e Hargreaves-Samani (HS) apresentaram coeficientes de desempenho de 84,05 e $79,52 \%$, respectivamente, enquanto os métodos Jensen \& Haise, Linacre e Makking apresentaram desempenho inferior a 60\%. A variação das condições de nebulosidade influenciaram o desempenho dos métodos de estimativa de ETo. Os melhores resultados foram obtidos pelos métodos da radiação solar $(86,1 \%)$ e de Camargo (81,8\%), nas condições Ab e Nub. Os métodos PMF e HS podem ser empregados de forma complementar, pois apresentaram desempenhos de 78,4\% (Nub e PNAd) e de 77,6\% (PNAb e Ab), respectivamente.

Termos para indexação: Hargreaves-Samani, lisímetro, manejo da irrigação, Penman-Monteith, transmissividade atmosférica.

\section{Reference evapotranspiration estimates in different cloudiness conditions}

\begin{abstract}
The objective of this work was to evaluate the performance of reference evapotranspiration (ETo) estimation methods, in different cloudiness conditions, in Seropédica, RJ, Brazil. The ETo estimates were compared with daily measurements made on weighing lysimeter, between 6/1/2006 and 7/31/2007, by pooling the data or discretizing them according to the variance of daily clearness index $\left(\mathrm{K}_{\mathrm{T}}\right)$, in four classes: $\mathrm{K}_{\mathrm{T}} \leq 0.35$, cloudy sky (Clo); $0.35<\mathrm{K}_{\mathrm{T}} \leq 0.55$, partly cloudy sky ( $\left.\mathrm{PCDi}\right) ; 0.55<\mathrm{K}_{\mathrm{T}} \leq 0.65$, partly opened (PCOp); and $\mathrm{K}_{\mathrm{T}}>0.65$, open sky $(\mathrm{Op})$. The mean absolute error (MBE), the square root of the mean square error (RMSE) and indexes of adjustment and performance were used as quality indicators of the different methods. In the pooled data, Penman-Monteith FAO (PMF) and Hargreaves-Samani (HS) methods had 84.05 and 79.52\% performance indexes, respectively, while Jensen \& Haise, Linacre and Makking methods had performances below $60 \%$. Changes in the cloudiness conditions affected the performance of ETo estimation methods. The best results were obtained with the solar radiation (86.1\%) and Camargo (81.8\%) methods, under the Op and Clo conditions. The PMF and HS methods can be employed in a complementary way, since their performance were $78.4 \%$ (Clo and PCDi) and 77.6\% (PCOp and Op), respectively.
\end{abstract}

Index terms: Hargreaves-Samani, lysimeter, irrigation management, Penman-Monteith, atmospheric transmissivity.

\section{Introdução}

A evapotranspiração constitui um dos principais parâmetros na estimativa do consumo de água pelas plantas e é definida como um processo conjunto e dinâmico de mudança do estado físico da água, dependente da disponibilidade de energia, da demanda atmosférica e do próprio suprimento de água do solo às plantas (Camargo \& Camargo, 2000; Kashyap \& Panda, 2001; Steduto et al., 2003).

A quantidade de energia disponível (saldo de radiação) é utilizada nos processos de evapotranspiração, fotossíntese e aquecimento do ar e do solo. Por conseguinte, sua variação exerce grande influência na perda de água pelo sistema solo-planta. De modo geral, se não houver restrição hídrica, a

Pesq. agropec. bras., Brasília, v.46, n.3, p.219-228, mar. 2011 
evapotranspiração é diretamente proporcional à disponibilidade de energia solar e ao saldo de radiação. Chang (1971) afirmou que a importância da radiação líquida, da umidade relativa do ar e da velocidade do vento sobre a evapotranspiração é de, aproximadamente, 80,6 e 4\%, respectivamente. A variação da quantidade de vapor d'água na atmosfera local normalmente está relacionada com a nebulosidade e influencia diretamente o balanço de ondas curtas e ondas longas que, por sua vez, altera o saldo de radiação. Assim, a forma de obtenção do saldo de radiação (Fietz \& Fisch, 2009) e a variação da nebulosidade podem afetar a evapotranspiração diária.

A evapotranspiração de referência (ETo) é definida pela perda de água de uma superfície extensa de solo, totalmente coberta por vegetação rasteira, com altura uniforme entre 8 e $15 \mathrm{~cm}$, em crescimento ativo e sem restrição hídrica (Doorenbos \& Pruitt, 1977; Allen et al., 1998). Entre os métodos utilizados na determinação da ETo, destaca-se o de lisímetros, que são estruturas especiais em que um volume de solo com vegetação é devidamente isolado, a fim de que todas as entradas e saídas de água sejam controladas. Carvalho et al. (2007) afirmaram que, para se obterem medidas precisas da evapotranspiração, a vegetação na área do lisímetro deve apresentar as mesmas condições (altura e área foliar semelhantes) da presente na área de contorno. Apesar da qualidade das informações obtidas, o uso de lisímetros somente se justifica em condições experimentais, com observações rigorosas das condições de contorno, para minimizar os efeitos advectivos, principalmente em época de deficiência hídrica.

A grande maioria dos métodos de estimação de ETo requer informações meteorológicas regionais. Esses métodos têm sido desenvolvidos e testados com emprego de diferentes elementos meteorológicos e agrupamentos de dados em diferentes centros de origem do modelo (Conceição \& Mandelli, 2005; Gavilán et al., 2006; Zanetti et al., 2008; Barros et al., 2009; Fietz \& Fischer, 2009; Trajkovic \& Kolakovic, 2009; Mendonça et al., 2010). O método de Penman-Monteith, parametrizado pela FAO (Allen et al., 1998), é considerado como padrão, por combinar componentes aerodinâmicos e de balanço de energia.

A seleção do método a ser empregado depende de fatores como condições climáticas, acessibilidade aos dados meteorológicos necessários, complexidade do método, agrupamento dos dados considerados e custos
(Carvalho et al., 2007; Zanetti et al., 2008). Desta forma, é recomendado que os diferentes modelos de estimativa sejam previamente avaliados e, se necessário, calibrados para as condições climáticas locais. Nesse processo, o desempenho desses modelos pode ser descrito por meio de índices de precisão e exatidão (Camargo \& Sentelhas, 1997; Gavilán et al., 2006; Barros et al., 2009).

O objetivo deste trabalho foi comparar o desempenho de nove métodos de estimativa da evapotranspiração de referência, em relação ao método de lisímetro, em diferentes condições de nebulosidade, no Município de Seropédica, RJ.

\section{Material e Métodos}

O estudo foi realizado com dados de evapotranspiração coletados no Sistema Integrado de Produção Agroecológica (Sipa), no Município de Seropédica, RJ (122 46 'S, $43^{\circ} 41^{\prime} \mathrm{W}$, a $33 \mathrm{~m}$ de altitude). Para a avaliação do comportamento da radiação solar na região, foram obtidos dados meteorológicos das estações de superfície convencional e automática, ambas pertencentes à rede do Instituto Nacional de Meteorologia (Inmet), localizadas a $22^{\circ} 45^{\prime} 13^{\prime \prime S}$ e 4340'23"W, também em Seropédica, RJ. Segundo a classificação de Köppen, o clima da região é do tipo Aw, com chuvas concentradas entre novembro e março, precipitação anual média de $1.213 \mathrm{~mm}$ e temperatura média anual de $23,9^{\circ} \mathrm{C}$ (Carvalho et al., 2006). $\mathrm{Na}$ Tabela 1, estão apresentadas as médias mensais de temperaturas e umidade relativa do ar, bem como radiação global e velocidade do vento observadas no município.

A regressão linear e o erro obtido na calibração da variação de massa, detectada pelo lisímetro de pesagem (Carvalho et al., 2007), estão apresentados na Figura 1. Próximo ao local de funcionamento do lisímetro, foram instalados sensores de radiação solar global incidente, de velocidade e direção do vento, e de temperatura e umidade relativa do ar, além de um pluviógrafo. Todos os sensores estavam interligados a um sistema de aquisição e armazenamento de dados, programado para coletar dados a cada três segundos, e as médias ou totais a cada 30 minutos.

Os dados coletados por esses sensores foram aplicados às comparações entre as estimativas da ETo por nove métodos: Penman-Monteith FAO 56, PMF; Hargreaves-Samani, HS; Camargo, CA; 
Priestley Taylor, PT; Makkink, MA; radiação solar FAO 24, RS; Linacre, LI; Jensen Haise, JH; e Turc, TU. O método do lisímetro de pesagem foi utlizado como padrão, para comparação com os métodos de estimação indireta. As comparações foram realizadas em diferentes condições de cobertura de céu, de acordo com os coeficientes de transmissividade da radiação global ou índices de claridade diários $\left(\mathrm{K}_{\mathrm{T}}^{\mathrm{d}}\right)$, definidos pela razão entre a radiação global e a radiação no topo da atmosfera. A classificação de cobertura de céu adotada seguiu a proposta de Escobedo et al. (2009): $\mathrm{K}_{\mathrm{T}}<0,35$, a radiação direta é praticamente nula, e a radiação global é igual à radiação difusa (céu nublado); $0,35 \leq \mathrm{K}_{\mathrm{T}}>0,55$, a radiação global é composta principalmente pela fração difusa e, em menor escala, pela radiação direta (céu parcialmente nublado); $0,55 \leq \mathrm{K}_{\mathrm{T}} \leq 0,65$, consiste no inverso do segundo intervalo, já que a radiação difusa diminui gradativamente (céu parcialmente aberto); e $\mathrm{K}_{\mathrm{T}}>0,65$, a radiação é composta principalmente pela radiação direta e mínima difusa (céu aberto). Segundo esses autores, para valores de $\left(\mathrm{K}_{\mathrm{T}}^{\mathrm{d}}\right)$ acima de 0,78 , não são verificadas sequências lógicas de evolução das três radiações em função da transmissividade atmosférica e, por essa razão, eles devem ser eliminados das análises.

Segundo as parametrizações propostas pela FAO (Allen et al, 1998), a equação original de Penman-Monteith assume a seguinte forma para determinação da ETo diária $\left(\mathrm{mm}\right.$ dia $\left.^{-1}\right)$ :

Tabela 1. Médias \pm desvio-padrão mensais de temperatura do ar, umidade relativa do ar, radiação global e velocidade do vento, de julho de 2000 a dezembro de 2006, em Seropédica, RJ.

\begin{tabular}{|c|c|c|c|c|c|c|}
\hline \multirow[t]{2}{*}{ Mês } & \multicolumn{3}{|c|}{ Temperatura do ar $\left({ }^{\circ} \mathrm{C}\right)$} & \multirow{2}{*}{$\begin{array}{c}\text { Umidade relativa } \\
\text { do } \operatorname{ar}(\%)\end{array}$} & \multirow{2}{*}{$\begin{array}{c}\text { Radiação global } \\
\left(\mathrm{MJ} \mathrm{m}^{-2} \text { dia }^{-1}\right)\end{array}$} & \multirow{2}{*}{$\begin{array}{c}\text { Velocidade } \\
\text { do vento }\left(\mathrm{m} \mathrm{s}^{-1}\right)\end{array}$} \\
\hline & Média & Máxima & Mínima & & & \\
\hline Janeiro & $26,42 \pm 1,09$ & $26,95 \pm 1,15$ & $25,90 \pm 1,06$ & $76,89 \pm 4,71$ & $20,77 \pm 3,87$ & $2,03 \pm 0,13$ \\
\hline Fevereiro & $26,42 \pm 1,47$ & $26,97 \pm 1,47$ & $25,86 \pm 1,39$ & $77,02 \pm 6,24$ & $20,86 \pm 3,93$ & $1,96 \pm 0,26$ \\
\hline Março & $26,08 \pm 0,99$ & $26,64 \pm 1,03$ & $25,53 \pm 0,95$ & $77,62 \pm 4,56$ & $18,77 \pm 2,12$ & $1,67 \pm 0,74$ \\
\hline Abril & $24,99 \pm 0,73$ & $25,55 \pm 0,76$ & $24,48 \pm 0,70$ & $78,75 \pm 3,49$ & $16,17 \pm 1,75$ & $1,52 \pm 0,75$ \\
\hline Maio & $21,62 \pm 1,02$ & $22,19 \pm 0,99$ & $21,09 \pm 1,03$ & $79,71 \pm 2,14$ & $12,91 \pm 1,09$ & $1,64 \pm 0,38$ \\
\hline Junho & $21,86 \pm 0,75$ & $22,48 \pm 0,77$ & $21,27 \pm 0,77$ & $75,33 \pm 3,12$ & $13,27 \pm 0,82$ & $2,03 \pm 0,18$ \\
\hline Julho & $21,06 \pm 1,09$ & $21,73 \pm 1,35$ & $20,56 \pm 1,29$ & $75,39 \pm 4,36$ & $12,10 \pm 1,36$ & $2,01 \pm 0,32$ \\
\hline Agosto & $22,03 \pm 1,48$ & $22,70 \pm 1,52$ & $21,42 \pm 1,43$ & $71,55 \pm 3,49$ & $14,46 \pm 1,59$ & $2,24 \pm 0,17$ \\
\hline Setembro & $21,79 \pm 0,22$ & $22,29 \pm 0,23$ & $21,32 \pm 0,21$ & $76,30 \pm 3,73$ & $14,12 \pm 1,80$ & $2,38 \pm 0,11$ \\
\hline Outubro & $25,19 \pm 3,97$ & $25,84 \pm 4,26$ & $24,65 \pm 3,91$ & $72,64 \pm 3,54$ & $18,03 \pm 1,52$ & $2,33 \pm 0,20$ \\
\hline Novembro & $24,29 \pm 0,46$ & $24,79 \pm 0,59$ & $23,82 \pm 0,54$ & $78,54 \pm 3,60$ & $17,69 \pm 1,47$ & $2,21 \pm 0,19$ \\
\hline$\underline{\text { Dezembro }}$ & $25,40 \pm 0,61$ & $25,91 \pm 0,60$ & $24,92 \pm 0,61$ & $78,63 \pm 3,05$ & $19,26 \pm 1,55$ & $2,14 \pm 0,22$ \\
\hline Ano & $23,93 \pm 2,03$ & $24,50 \pm 2,01$ & $23,40 \pm 2,03$ & $76,53 \pm 2,60$ & $16,53 \pm 2,95$ & $2,01 \pm 0,29$ \\
\hline
\end{tabular}
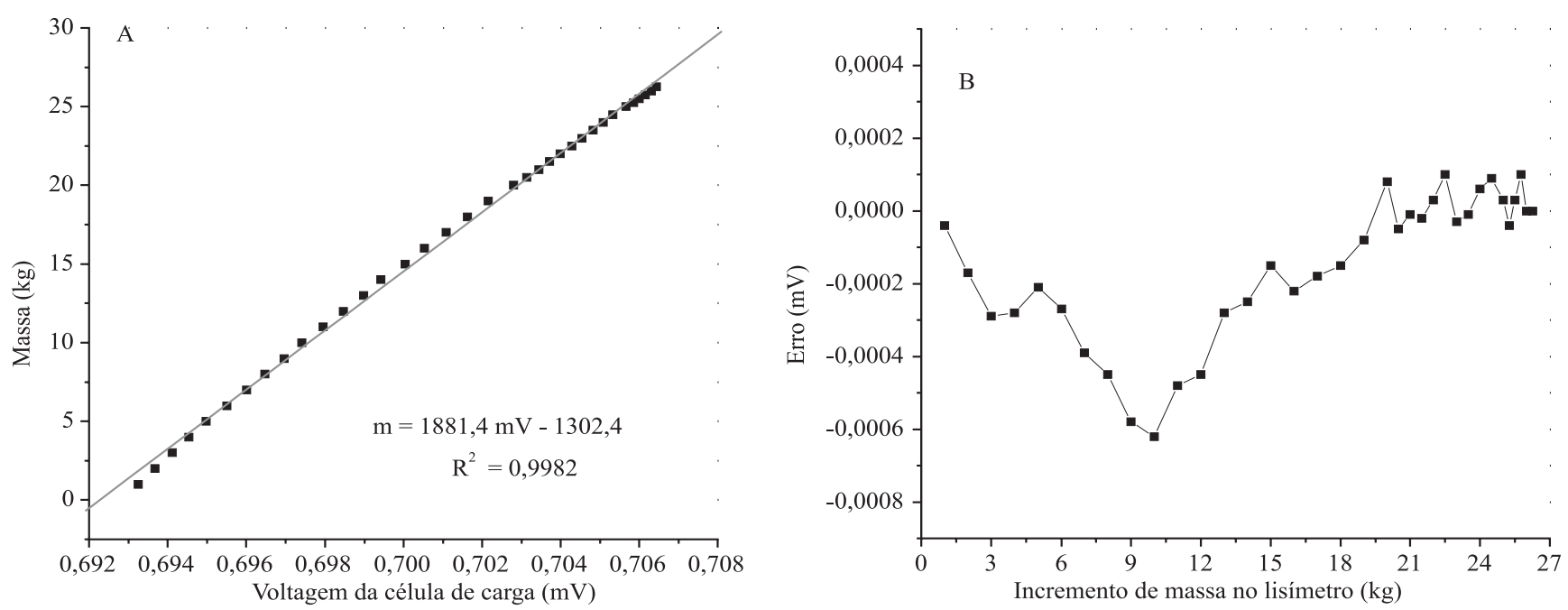

Figura 1. Correlação entre a voltagem da célula de carga e a variação de massa medida no lisímetro (A), e erro resultante do incremento de massa no lisímetro de pesagem (B). 


$$
\begin{aligned}
& \mathrm{ET}_{0}=\left[0,408 \cdot \mathrm{S} \cdot\left(\mathrm{R}_{\mathrm{N}}-\mathrm{G}\right)+\gamma \cdot \frac{900}{\mathrm{~T}+273} \cdot \mathrm{u}_{2} \cdot\left(\mathrm{e}_{\mathrm{s}}-\mathrm{e}_{\mathrm{a}}\right)\right] \\
& /\left[\mathrm{S}+\gamma \cdot\left(1+0,34 \cdot \mathrm{u}_{2}\right)\right]
\end{aligned}
$$

em que: Rn é o saldo de radiação total diário $\left(\mathrm{MJ} \mathrm{m}^{-2}\right.$ dia $\left.^{-1}\right)$; $\mathrm{G}$ é a densidade de fluxo de calor no solo (MJ $\mathrm{m}^{-2}$ por dia); $\mathrm{T}$ é a temperatura média diária do ar a $2 \mathrm{~m}$ de altura $\left({ }^{\circ} \mathrm{C}\right) ; \mathrm{u}_{2}$ é a velocidade do vento média diária a $2 \mathrm{~m}$ de altura $\left(\mathrm{m} \mathrm{s}^{-1}\right)$; $\mathrm{e}_{\mathrm{s}}$ é a pressão de saturação do vapor média diária $(\mathrm{kPa})$; $\mathrm{e}_{\mathrm{a}}$ é a pressão parcial de vapor média diária $(\mathrm{kPa})$; $\mathrm{S}$ é a declividade da curva de pressão de saturação de vapor, no ponto da temperatura média $\left(\mathrm{kPa}^{\circ} \mathrm{C}^{-1}\right)$; e $\gamma$ é o coeficiente psicrométrico $\left(\mathrm{kPa}^{\circ} \mathrm{C}^{-1}\right)$. Os valores de $\mathrm{S}$ e $\gamma$ foram calculados pela metodologia apresentada por Allen et al. (1998). A radiação líquida $\left(\mathrm{R}_{\mathrm{N}}\right)$ foi estimada pela soma do saldo de radiação de ondas curtas com o saldo de radiação de ondas longas, que também foram calculados de acordo com Allen et al. (1998).

O método de Hargreaves-Samani, descrito por Pereira et al. (1997) e Samani (2000) é dado por $\mathrm{ETo}=0,0023 \mathrm{Q}_{0}\left(\mathrm{~T}_{\text {máx }}-\mathrm{T}_{\text {mín }}\right)^{0,5}(\mathrm{~T}+17,8)$, em que: $\mathrm{Q}_{0}$ é a radiação no topo da atmosfera, expressa em equivalente de evaporação $\left(\mathrm{MJ} \mathrm{m}^{-2}\right.$ dia $\left.^{-1}\right)$; T é a temperatura do ar média diária a $2 \mathrm{~m}$ de altura $\left({ }^{\circ} \mathrm{C}\right) ; \mathrm{T}_{\text {máx }}$ é a temperatura do ar máxima diária a $2 \mathrm{~m}$ de altura $\left({ }^{\circ} \mathrm{C}\right)$; e $\mathrm{T}_{\min }$ é a temperatura do ar mínima diária a $2 \mathrm{~m}$ de altura $\left({ }^{\circ} \mathrm{C}\right)$.

$\mathrm{O}$ método de Camargo varia com a temperatura média do período considerado e com a radiação no topo da atmosfera, e é descrito por ETo $=\mathrm{F}_{0} \mathrm{~T} \mathrm{ND}$, em que: $\mathrm{F}$ é o fator de ajuste, que varia com a temperatura média anual do ar (Ta) - foi empregado um valor de $F$ igual a 0,0105 , correspondente a um valor de Ta de até $23^{\circ} \mathrm{C}$.

O método de Makkink (1957) correlaciona a evapotranspiração e radiação solar na superfície, conforme Pereira et al. (1997), na equação $\mathrm{ETo}=0,61 \mathrm{~W} \mathrm{Q}_{0}-0,12$, em que: $\mathrm{W}=\mathrm{S} /(\mathrm{S}+\gamma)$ é um fator de ponderação dependente da temperatura do bulbo úmido e do coeficiente psicrométrico.

O método de Priestley \& Taylor (1972) pode ser considerado como um método de balanço de energia baseado apenas no termo radiativo (diabático) corrigido por um fator de ajuste que torna o método semelhante à razão de Bowen (Pereira et al., 1997): $\mathrm{ET}_{0}=1,26 \mathrm{~W}$ $\left(R_{N}-G\right)$, cujas variáveis já foram apresentadas acima.

$\mathrm{O}$ método da radiação solar, também conhecido como método FAO 24 da radiação, se tornou uma adaptação do método de Makkink, com o incremento de um coeficiente dependente da umidade relativa do ar e da velocidade do vento, traduzido como coeficiente angular da regressão $\mathrm{ET}_{0} \times \mathrm{W} \mathrm{R}_{\mathrm{G}}$ (Pereira et al., 1997) em $\mathrm{ETo}=\mathrm{cW} \mathrm{R}$, em que: $\mathrm{R}_{\mathrm{G}}$ é a radiação solar global, expressa em equivalente de evaporação $\left(\mathrm{mm} \mathrm{dia}^{-1}\right)$.

A evapotranspiração obtida pelo método de Linacre (1977) considera a influência da altitude, da latitude e das temperaturas diárias máxima, mínima e do ponto de orvalho, de acordo com a seguinte equação:

$$
\mathrm{ET}_{0}=\left[700 \frac{\mathrm{T}_{\mathrm{m}}}{(100-\Phi)}+15\left(\mathrm{~T}-\mathrm{T}_{\mathrm{d}}\right)\right] /\left(80-\mathrm{T}_{\mathrm{a}}\right),
$$

em que: $\mathrm{T}_{\mathrm{m}}$ é a temperatura média diária do ar, em ${ }^{\circ} \mathrm{C} ; \mathrm{z}$ é a altitude em metros - neste caso, $\mathrm{T}_{\mathrm{m}}=\mathrm{T}_{\mathrm{a}}+0,006 \mathrm{z} ; \Phi$ é a latitude local, em graus; $T_{d}$ é a temperatura de ponto de orvalho, em ${ }^{\circ} \mathrm{C}$.

O método de Jensen \& Haise (1963) apresenta aplicações para regiões áridas e semiáridas, com a radiação global ao nível do solo expressa, em equivalente de evaporação $\left(\mathrm{mm} \mathrm{dia}{ }^{-1}\right)$, por $\mathrm{ETo}=\mathrm{R}_{\mathrm{G}}(0,0252 \mathrm{~T}+0,078)$.

O método de Turc (1961) foi desenvolvido para o Oeste europeu, onde a umidade relativa do ar é maior que $50 \%$, e tem sido aplicado em diferentes regiões. A equação para a estimativa da ETo foi descrita por Kashyap \& Panda (2001) como:

$\mathrm{ET}_{0}=\mathrm{a}_{\mathrm{t}} \cdot 0,013 \cdot\left\{\mathrm{T}_{\text {med }} /\left(\mathrm{T}_{\text {med }}+15\right) \cdot\left[\left(\frac{\mathrm{R}_{\mathrm{G}}}{0,0238846}+50\right) / \lambda\right]\right\}$,

em que: $R_{\mathrm{G}}$ é a radiação solar global, em $\mathrm{MJ} \mathrm{m}^{-2}$ $\mathrm{dia}^{-1} ; \lambda$ é o calor latente de vaporização $\left(\mathrm{MJ} \mathrm{mm}^{-1}\right)$; o coeficiente $\mathrm{a}_{\mathrm{t}}$ tem como base a umidade relativa do ar (UR), quando a média diária de UR é maior ou igual a $50 \%, a_{t}=1,0$, caso contrário $a_{t}=1+((U R-50) / 70)$.

As comparações entre estimativas e medidas da ETo foram realizadas no período de junho de 2006 a julho de 2007, com dados medidos na estação agrometeorológica do Sipa. Em ambas as estações, esses dados sofreram análise de consistência, para eliminar erros de medidas causadas principalmente por problemas elétricos nos radiômetros e nas células de carga.

As avaliações dos desempenhos dos modelos de estimativa de ETo, nas diferentes condições de cobertura de céu, foram baseadas nos indicadores estatísticos erro absoluto médio ("mean bias error", 
$\mathrm{MBE}$ ), raiz quadrada do quadradro médio do erro ("root mean square error", RMSE), e índice de ajustamento d, conforme Escobedo et al. (2009). Determinouse também o índice de desempenho c (Camargo \& Sentelhas, 1997), correspondente ao produto entre o coeficiente de correlação r e o índice de ajustamento d. Os indicadores estatísticos foram calculados de acordo com as seguintes equações:

$$
\begin{aligned}
\mathrm{MBE} & =\sum_{\mathrm{i}=1}^{\mathrm{N}}(\mathrm{Pi}-\mathrm{Oi}) / \mathrm{N}, \quad \mathrm{RMSE}=\left[\sum_{\mathrm{i}=1}^{\mathrm{N}}(\mathrm{Pi}-\mathrm{Oi})^{2} / \mathrm{N}\right]^{\frac{1}{2}}, \\
\mathrm{e} d & =1-\left[\sum_{\mathrm{i}=1}^{\mathrm{N}}(\mathrm{Pi}-\mathrm{Oi})^{2} / \sum_{\mathrm{i}=1}^{\mathrm{N}}\left(\left|\mathrm{P}^{\prime} \mathrm{i}\right|+\left|\mathrm{O}^{\prime} \mathrm{i}\right|\right)^{2}\right],
\end{aligned}
$$

em que: Pi representa os valores estimados; Oi são os valores medidos; $\mathrm{N}$ é o número de observações; $\left|\mathrm{P}^{\prime} \mathrm{i}\right|$ é o valor absoluto da diferença P - $\overline{\mathrm{O}}^{\prime}$; $\left|\mathrm{O}^{\prime} \mathrm{i}\right|$ é o valor absoluto da diferença Oi -Ōi.

O índice de desempenho, normalmente, é distribuído nas seguintes classes: péssimo, $\mathrm{c} \leq 0,40$; mau, $0,41 \leq \mathrm{c} \leq 0,50$; sofrível, $0,51 \leq \mathrm{c} \leq 0,60$; mediano, $0,61 \leq \mathrm{c} \leq 0,65$; bom, $0,66 \leq \mathrm{c} \leq 0,75$; muito bom, $0,76 \leq \mathrm{c} \leq 0,85$; e ótimo, $\mathrm{c}>0,85$ (Camargo \& Sentelhas, 1997).

O indicador MBE representa o desvio das médias e provê informações quanto ao desempenho do modelo a longo prazo, com valores negativos indicando subestimativa e vice-versa. Quanto menor o valor absoluto de MBE, melhor é o desempenho do modelo

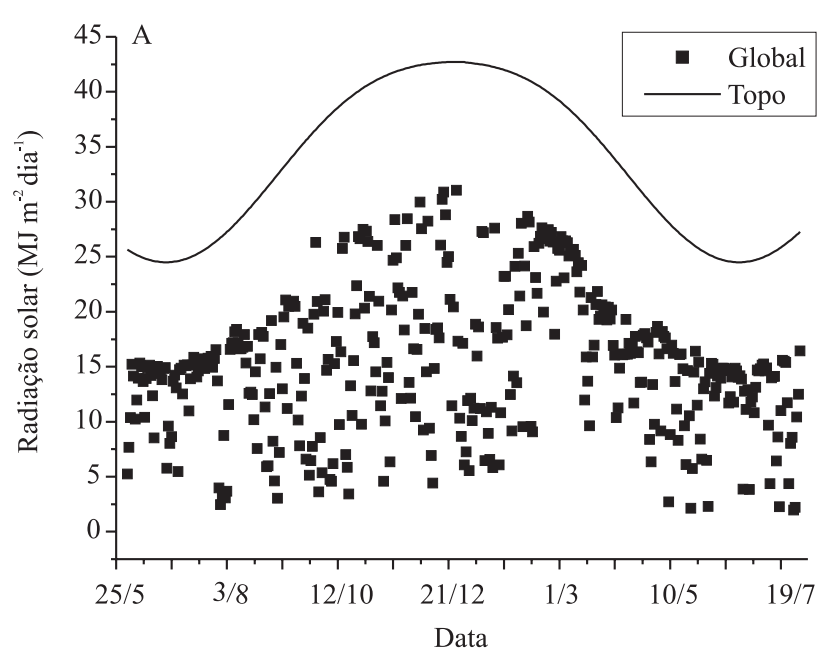

testado (Stone, 1993), porém, uma superestimativa cancela uma subestimativa. O RMSE informa sobre o valor real do erro produzido pelo modelo. Todavia, alguns erros de grande proporção na soma podem causar acréscimos significativos aos valores de RMSE. O índice de ajustamento $\mathrm{d}$ varia de 0 a 1 e representa o quanto os valores estimados se ajustam aos valores medidos.

A utilização dos indicadores estatísticos MBE, RMSE, índice de ajustamento d e índice de desempenho c, em conjunto, permite uma alternativa adequada para validação de modelos estatísticos, com análises simultâneas dos desvios da média.

\section{Resultados e Discussão}

A evolução das radiações diárias globais $\left(\mathrm{H}_{\mathrm{G}}^{\mathrm{d}}\right)$ e no topo da atmosfera $\left(\mathrm{H}_{0}^{\mathrm{d}}\right)$, entre 2006 e 2007 , apresentou características sazonais da transmissão da radiação global na atmosfera local, com valores máximos no verão e mínimos no inverno (Figura 2). A distribuição de frequência diária de $\mathrm{K}_{\mathrm{T}}$ foi do tipo bimodal, com intervalo de variação entre 0,0733 e 0,7266 , com o maior número de frações (149 dias, ou 35,06\%) entre 0,55 e 0,65 . Cerca de $53,19 \%$ dos dias apresentaram transmissividade atmosférica acima de $50 \%$, o que indica que a região apresentou cobertura de céu tendendo à aberta durante o período.

Todas as análises de regressão foram realizadas, tendo-se considerado o coeficiente linear igual a zero (Tabela 2), embora a tendência natural seja a de que a

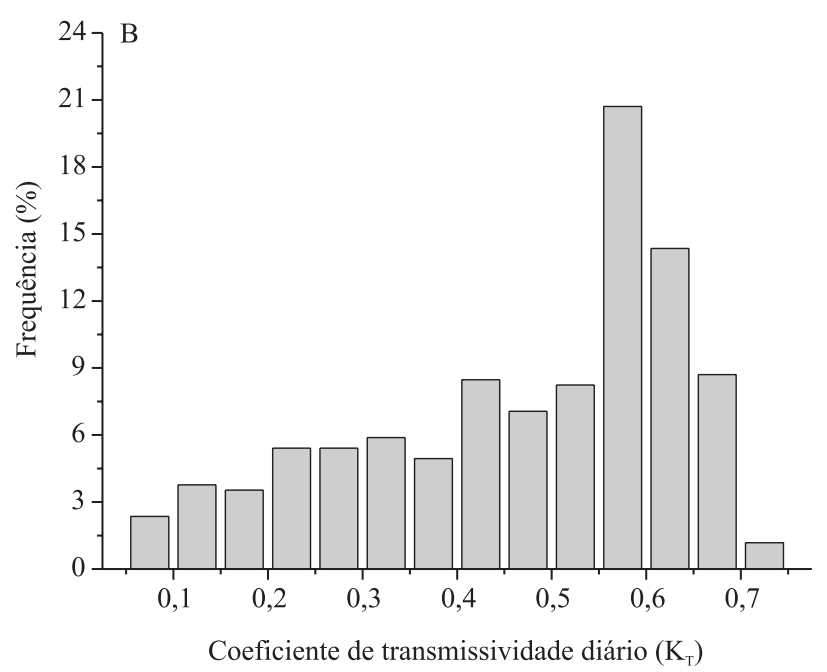

Figura 2. Evolução diária da radiação global e no topo da atmosfera $(\mathrm{A})$, e frequência de ocorrência dos coeficientes diários de transmissividade global (B), entre junho 2006 e julho de 2007, em Seropédica, RJ. 
ETo estimada apenas se aproxime de zero à medida que a contribuição da temperatura diminua e a da umidade relativa aumente. Coeficientes lineares nulos, no entanto, garantem a inexistência de resíduos na estimativa, quando a ETo medida é zero (Barros et al., 2009).

Com o agrupamento dos dados, apenas o método de TU apresentou valores de ETo menores do que os valores medidos no lisímetro de pesagem, o que foi mostrado pelo coeficiente angular maior que 1 . A maioria dos modelos nesse agrupamento apresentou tendência a subestimar os valores de ETo, com exceção dos modelos CA, LI e PT, cujas superestimativas foram inferiores a $0,96 \mathrm{~mm} \mathrm{dia}^{-1}$. Esses métodos ainda apresentaram, em conjunto com TU, os maiores espalhamentos entre dados estimados e medidos, com valores que chegaram a $1,50 \mathrm{~mm} \mathrm{dia}^{-1}$.

O modelo PMF parametrizado pela FAO foi o que apresentou as estimativas mais satisfatórias da ETo, em razão da pequena dispersão dos pontos, descrita pelo coeficiente angular de 0,93 e coeficiente de correlação de 0,9139. Trajkovic \& Kolakovic (2009) e Barros et al. (2009) também verificaram a superioridade desse modelo, pelo melhor desempenho observado em todos os indicadores estatísticos empregados.

O desempenho muito bom, obtido no presente trabalho pelo método de HS, já foi verificado em algumas regiões brasileiras, o que o torna uma boa alternativa quando há ausência de elementos meteorológicos necessários à aplicação do método PMF (Conceição \& Mandelli, 2005; Barros et al., 2009). Pelos resultados encontrados no agrupamento total, o método HS apresentou tendência em subestimar os valores de ETo medidos no lisímetro, em torno de $0,02 \mathrm{~mm} \mathrm{dia}^{-1}$, com espalhamentos de $0,13 \mathrm{~mm} \mathrm{dia}^{-1}$; porém, esse método apresentou coeficientes de correlação inferiores aos obtidos com PMF.

Outra possibilidade seria a aplicação do método da radiação solar, cujos resultados encontrados corroboraram os de Conceição \& Mandelli (2005) e Mendonça \& Dantas (2010). De acordo com esses autores, as comparações entre valores estimados deETo, por métodos que empregam a radiação solar incidente, normalmente apresentam desempenhos superiores aos que utilizam apenas valores de temperatura do ar.

Com relação aos métodos MA, JH e LI, os ajustes apresentaram os mais baixos coeficientes de desempenho, o que os torna não recomendáveis para estimativas diárias de ETo, na região de Seropédica, RJ. Esse comportamento foi influenciado pela origem do método, visto que, segundo Camargo \& Camargo (2000), eles são indicados para estimativa da evapotranspiração em períodos agrupados de 10 ou 30 dias. Todavia, Barros et al. (2009) verificaram bons resultados com a aplicação desse método em agrupamentos de dados de três dias.

Quando o agrupamento dos dados foi discretizado em função da cobertura do céu, o comportamento da estimativa do modelo PMF foi alterada (Figura 3). $\mathrm{Na}$ condição de céu nublado, com baixos saldos de radiação disponíveis, o processo de evapotranspiração se torna dependente principalmente da interação aerodinâmica entre planta e atmosfera e apresenta redução dos níveis de ETo. Contudo, com o céu nublado, o lisímetro de pesagem registrou valores de ETo superiores aos estimados por PMF.

Tabela 2. Coeficientes angulares das regressões entre os valores diários medidos no lisímetro de pesagem (LIS) e os estimados por diferentes métodos, com seus respectivos indicadores de desempenho, com o uso do agrupamento total de dados.

\begin{tabular}{|c|c|c|c|c|c|c|c|}
\hline \multirow[t]{2}{*}{ Modelo $^{(1)}$} & \multirow[t]{2}{*}{ Coeficiente angular } & \multicolumn{5}{|c|}{ Indicador de desempenho ${ }^{(2)}$} & \multirow[t]{2}{*}{ Classe } \\
\hline & & $\operatorname{MBE}\left(\mathrm{mm} \mathrm{dia}{ }^{-1}\right)$ & RMSE $\left(\mathrm{mm} \mathrm{dia}{ }^{-1}\right)$ & $\mathrm{d}$ & $\mathrm{r}$ & $\mathrm{c}$ & \\
\hline PMF x LIS & 0,93112 & $-0,5910$ & 0,6283 & 0,9197 & 0,9139 & 0,8405 & Muito bom \\
\hline CA $x$ LIS & 0,62250 & 0,9528 & 1,4979 & 0,6953 & 0,8959 & 0,6229 & Mediano \\
\hline HS x LIS & 0,67304 & $-0,0173$ & 0,1280 & 0,9968 & 0,7977 & 0,7952 & Muito bom \\
\hline JH x LIS & 0,39615 & $-0,5958$ & 0,8456 & 0,8412 & 0,7033 & 0,5916 & Sofrível \\
\hline LI x LIS & 0,65000 & 0,9231 & 1,3258 & 0,6871 & 0,7619 & 0,5235 & Sofrível \\
\hline MA $x$ LIS & 0,45550 & $-0,3937$ & 0,7926 & 0,8166 & 0,6989 & 0,5707 & Sofrível \\
\hline PT x LIS & 0,41385 & 0,2693 & 0,8398 & 0,8125 & 0,7462 & 0,6063 & Mediano \\
\hline RS $x$ LIS & 0,74654 & $-0,3961$ & 0,7166 & 0,8565 & 0,8332 & 0,7136 & Bom \\
\hline TU $x$ LIS & 1,12972 & $-0,8611$ & 1,1104 & 0,7077 & 0,8742 & 0,6187 & Mediano \\
\hline
\end{tabular}

(1)PMF, Penman-Monteith FAO; CA, Camargo; HS, Hargreaves-Samani; JH, Jensen Haise; LI, Linacre; MA, Makkink; PT, Priestley Taylor; RS, radiação solar FAO 24; TU, Turc. (2) MBE, erro absoluto médio; RMSE, raiz quadrada do quadrado médio do erro; d, índice de desempenho; r, coeficiente de correlação linear; c, índice de ajustamento. 
Nesses agrupamentos de dados, o método PMF apresentou coeficientes angulares de 1,1084, 0,9253, 0,9095 e 0,9237 , com coeficientes de correlação iguais a $0,7876,0,8386,0,8297$ e 0,8655 , respectivos às condições de céu nublado, parcialmente nublado, parcialmente aberto e aberto. Foram observadas tendências de subestimativas apenas para o agrupamento com céu nublado $\left(-0,039 \mathrm{~mm} \mathrm{dia}^{-1}\right)$, e os maiores espalhamentos ocorreram nos dados relativos a coberturas de céu de transição (máximo de $\left.0,457 \mathrm{~mm} \mathrm{dia}^{-1}\right)$. Os índices de desempenho foram de $78,43,79,33,73,75$ e $66,48 \%$, respectivamente para céu nublado, parcialmente nublado, parcialmente aberto e aberto. Quando a cobertura de céu diminuiu, observou-se que as correlações entre valores medidos e estimados melhoraram, porém os desempenhos pioraram em razão do aumento dos desvios. Gomide \& Maeno (2008) verificaram que, em geral, o modelo PMF indica superestimativas de 8 a $35 \%$ dos valores medidos em lisímetro, de acordo com o clima da região. Esse método apresenta subestimativas em casos de elevadas taxas de ETo (Steduto et al., 2003).

O bom desempenho do método HS, para condições de céu aberto (Tabela 3), está de acordo com o fato de esse método ter sido ajustado para as condições semiáridas da Califórnia (Pereira et al., 1997; Samani, 2000). Nas condições de céu nublado, o seu desempenho foi decorrente das pequenas diferenças observadas entre temperaturas máxima e mínima, que influenciaram as condições de umidade do ar na região (Samani, 2000). $\mathrm{O}$ método HS apresentou tendência de subestimar os valores de ETo em condições de céu parcialmente aberto (cerca de $-0,19 \mathrm{~mm} \mathrm{dia}^{-1}$ ), o que corrobora os resultados de Oliveira et al. (2001) e Gavilán et al. (2006), que verificaram piores desempenhos em períodos chuvosos.

Trajkovic \& Kolakovic (2009) obtiveram, para condições de clima úmido, espalhamentos nos valores
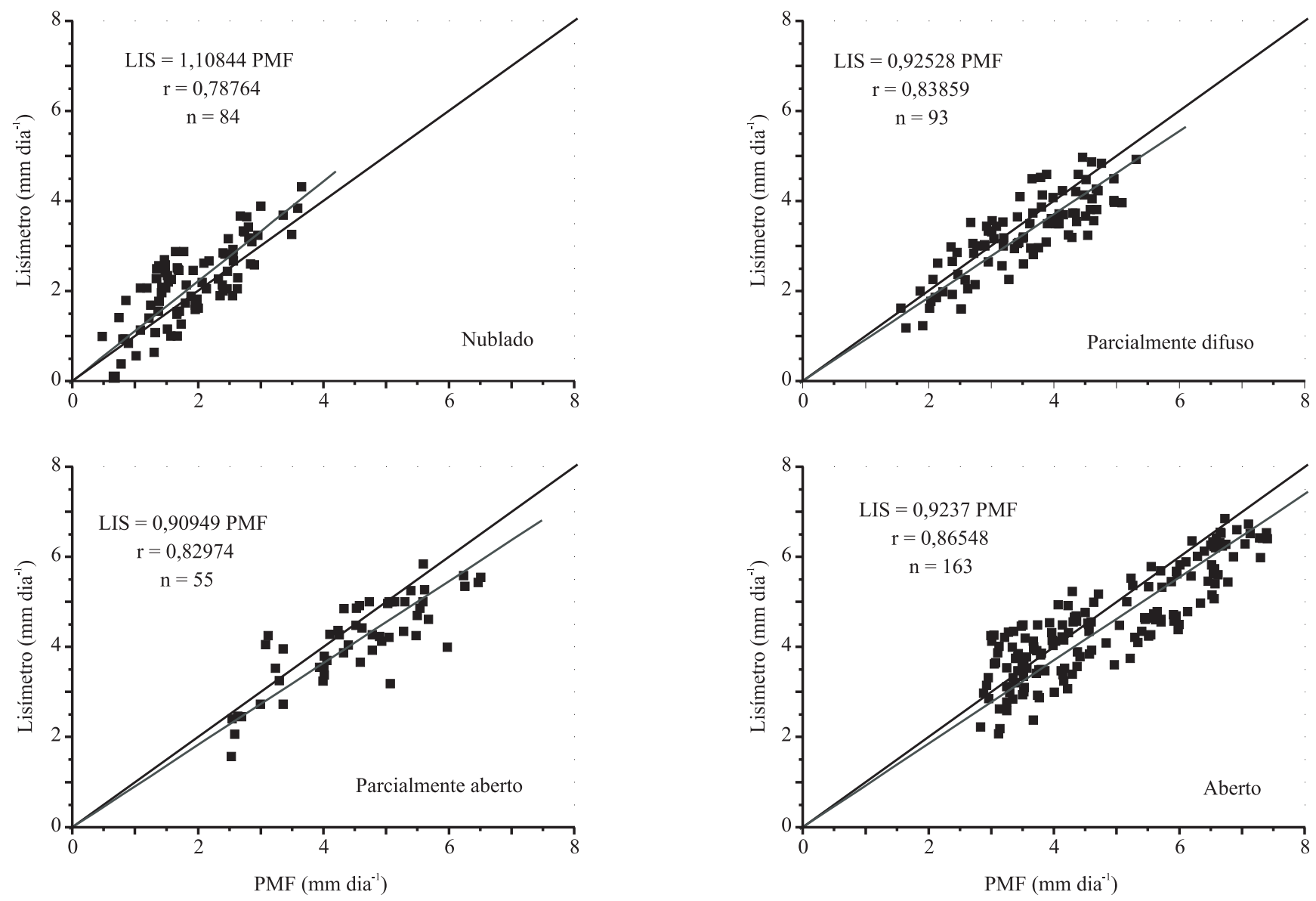

Figura 3. Correlação entre valores de evapotranspiração de referência ( $\mathrm{mm} \mathrm{dia}^{-1}$ ) estimados pelo método Penman-Monteith FAO 56 (PMF) e os medidos no lisímetro de pesagem (LIS), em diferentes condições de cobertura de céu. 
de ETo, estimados por HS e PMF, que variaram entre 0,30 e $0,90 \mathrm{~mm} \mathrm{dia}^{-1}$. No método HS, a ETo se baseia na estimativa da radiação global com base em $\mathrm{Q}_{0}$ e na diferença entre as temperaturas máxima e mínima do ar. Assim, em presença de nuvens, quando os valores de $\mathrm{R}_{\mathrm{G}}$ são menores, a amplitude térmica também tende a ser menor (Hargreaves \& Allen, 2003; Conceição, 2010).

Os métodos MA e TU subestimaram a ETo medida no lisímetro, nas quatro condições de cobertura de céu, com variações de até $0,39 \mathrm{~mm} \mathrm{dia}^{-1}$. Vescove \& Turco (2005) verificaram que o método MA subestimou a ETo estimada por PMF, principalmente nos meses de inverno-primavera, o que está de acordo com os resultados encontrados no presente trabalho.

$\mathrm{O}$ método $\mathrm{CA}$, discretizado de acordo com as diferentes nebulosidades, apresentou bons resultados para coberturas de céu com maiores nebulosidades (coeficiente de desempenho de $81,8 \%$ ), o que

Tabela 3. Coeficientes angulares das regressões entre os valores diários medidos no lisímetro de pesagem (LIS) e os estimados por diferentes métodos, com seus respectivos indicadores de desempenho, em diferentes condições de cobertura de céu.

\begin{tabular}{|c|c|c|c|c|c|c|c|c|}
\hline \multirow[t]{2}{*}{ Correlação $o^{(1)}$} & \multirow[t]{2}{*}{ Cobertura de céu } & \multirow{2}{*}{$\begin{array}{c}\text { Coeficiente } \\
\text { angular }\end{array}$} & \multicolumn{5}{|c|}{ Indicador de desempenho $^{(2)}$} & \multirow[t]{2}{*}{ Classe } \\
\hline & & & $\operatorname{MBE}\left(\mathrm{mm} \mathrm{dia}^{-1}\right)$ & $\operatorname{RMSE}\left(\mathrm{mm} \operatorname{dia}^{-1}\right)$ & $\mathrm{d}$ & $\mathrm{r}$ & $\mathrm{c}$ & \\
\hline \multirow{4}{*}{ PMF x LIS } & Nublado & 1,10844 & $-0,039$ & 0,131 & 0,9958 & 0,7876 & 0,7843 & Muito Bom \\
\hline & Parc. difuso & 0,92528 & 0,032 & 0,412 & 0,9460 & 0,8386 & 0,7933 & Muito Bom \\
\hline & Parc. aberto & 0,90949 & 0,121 & 0,457 & 0,8888 & 0,8297 & 0,7375 & Bom \\
\hline & Aberto & 0,9237 & 0,214 & 0,367 & 0,7682 & 0,8655 & 0,6648 & Mediano \\
\hline \multirow{4}{*}{ CA $x$ LIS } & Nublado & 0,58770 & 0,3998 & 0,5869 & 0,9143 & 0,8952 & 0,818 & Muito Bom \\
\hline & Parc. difuso & 0,58850 & 0,8375 & 1,5064 & 0,6963 & 0,8444 & 0,588 & Sofrível \\
\hline & Parc. aberto & 0,65004 & 0,6544 & 0,8847 & 0,7034 & 0,8586 & 0,604 & Sofrível \\
\hline & Aberto & 0,63618 & 0,5088 & 0,5247 & 0,7703 & 0,8651 & 0,666 & Bom \\
\hline \multirow{4}{*}{ HS $x$ LIS } & Nublado & 0,66253 & 0,0130 & 0,5671 & 0,881 & 0,8295 & 0,731 & Bom \\
\hline & Parc. difuso & 0,65498 & 0,0965 & 1,1283 & 0,779 & 0,8222 & 0,640 & Mediano \\
\hline & Parc. aberto & 0,67842 & $-0,1934$ & 0,4385 & 0,941 & 0,8237 & 0,776 & Muito bom \\
\hline & Aberto & 0,70733 & 0,0135 & 0,0252 & 0,999 & 0,7791 & 0,779 & Muito bom \\
\hline \multirow{4}{*}{ JH x LIS } & Nublado & 0,68997 & 1,5313 & 1,6930 & 0,6807 & 0,8351 & 0,569 & Sofrível \\
\hline & Parc. difuso & 0,46242 & 0,9250 & 1,0907 & 0,7936 & 0,7783 & 0,618 & Mediano \\
\hline & Parc. aberto & 0,37241 & 0,7455 & 0,8202 & 0,8052 & 0,8496 & 0,684 & Bom \\
\hline & Aberto & 0,28141 & 0,0928 & 0,3063 & 0,8304 & 0,8525 & 0,708 & Bom \\
\hline \multirow{4}{*}{ LI x LIS } & Nublado & 0,46259 & 0,6173 & 0,7682 & 0,8448 & 0,7897 & 0,667 & Mediano \\
\hline & Parc. difuso & 0,58972 & 0,2588 & 0,4980 & 0,9350 & 0,6568 & 0,614 & Mediano \\
\hline & Parc. aberto & 0,70163 & 0,3768 & 0,6170 & 0,8655 & 0,7796 & 0,675 & Bom \\
\hline & Aberto & 0,71688 & $-0,3933$ & 0,4172 & 0,8198 & 0,8446 & 0,692 & Bom \\
\hline \multirow{4}{*}{ MA x LIS } & Nublado & 0,57488 & $-0,3561$ & 0,6927 & 0,8268 & 0,7483 & 0,619 & Mediano \\
\hline & Parc. difuso & 0,45713 & $-0,2228$ & 0,6415 & 0,8440 & 0,8308 & 0,701 & Bom \\
\hline & Parc. aberto & 0,43973 & $-0,2074$ & 0,5141 & 0,8646 & 0,7807 & 0,675 & Bom \\
\hline & Aberto & 0,45047 & $-0,2886$ & 0,4685 & 0,6710 & 0,8051 & 0,540 & Sofrível \\
\hline \multirow{4}{*}{ PT $x$ LIS } & Nublado & 0,58143 & 0,6325 & 0,9003 & 0,7856 & 0,8964 & 0,704 & Bom \\
\hline & Parc. difuso & 0,44373 & 0,6923 & 0,9768 & 0,7423 & 0,8271 & 0,614 & Mediano \\
\hline & Parc. aberto & 0,44919 & 1,2980 & 1,3216 & 0,6552 & 0,8131 & 0,533 & Sofrível \\
\hline & Aberto & 0,39172 & 0,5726 & 0,6556 & 0,6480 & 0,8655 & 0,561 & Sofrível \\
\hline \multirow{4}{*}{ RS $x$ LIS } & Nublado & 0,95373 & $-0,2968$ & 0,4864 & 0,9279 & 0,7824 & 0,726 & Bom \\
\hline & Parc. difuso & 0,77588 & $-0,1128$ & 0,5107 & 0,9073 & 0,8433 & 0,765 & Muito Bom \\
\hline & Parc. aberto & 0,74035 & $-0,1541$ & 0,5099 & 0,8555 & 0,8297 & 0,710 & Bom \\
\hline & Aberto & 0,72377 & 0,0718 & 0,0742 & 0,9947 & 0,8655 & 0,861 & Ótimo \\
\hline \multirow{4}{*}{ TU x LIS } & Nublado & 1,62634 & $-0,3894$ & 0,7149 & 0,8089 & 0,7897 & 0,639 & Mediano \\
\hline & Parc. difuso & 1,36126 & $-0,1735$ & 0,6201 & 0,8471 & 0,8326 & 0,705 & Bom \\
\hline & Parc. aberto & 1,15269 & $-0,3174$ & 0,5076 & 0,9298 & 0,7897 & 0,734 & Bom \\
\hline & Aberto & 1,05523 & $-0,3933$ & 0,4172 & 0,8198 & 0,8446 & 0,692 & Bom \\
\hline
\end{tabular}

(1)PMF, Penman-Monteith FAO 56; CA, Camargo; HS, Hargreaves-Samani; JH, Jensen Haise; LI, Linacre; MA, Makkink; PT, Priestley Taylor; RS, radiação solar FAO 24; TU, Turc. ${ }^{(2)} \mathrm{MBE}$, erro absoluto médio; RMSE, raiz quadrada do quadrado médio do erro; d, índice de desempenho; r, coeficiente de correlação linear; c, índice de ajustamento. 
permite sua recomendação. Com o céu parcialmente nublado, parcialmente aberto e aberto, os melhores indicadores foram encontrados com os métodos RS e HS. O método RS propiciou desempenhos de 76,5 e $86,1 \%$, classificados como muito bom e ótimo, respectivamente. $\mathrm{O}$ método $\mathrm{PT}$ apresentou tendências de superestimar a evapotranspiração, medida no lisímetro em todas as condições de nebulosidade, com diminuição do desempenho em condições de céu aberto, o que está de acordo com os resultados de Fietz \& Fisch (2009). Por sua vez, o modelo $\mathrm{JH}$, que foi desenvolvido para condições áridas e semiáridas (Pereira et al., 1997), apresentou melhoria do desempenho quando a transmissividade atmosférica aumentou. A equação proposta por Linacre (1977) apresentou o mesmo comportamento do método $\mathrm{JH}$, embora não seja originária das mesmas condições climáticas e não considere o efeito da radiação solar global. Mendonça \& Dantas (2010) verificaram que o método LI apresentou piores ajustes durante os dias de verão, que normalmente são caracterizados por grande variação da nebulosidade local.

\section{Conclusões}

1. Os métodos de Penman-Monteith FAO e Hargreaves-Samani são eficazes para a estimativa da evapotranspiração de referência (ETo), nas condições climáticas de Seropédica, RJ.

2. A variação da nebulosidade influencia o desempenho dos métodos de estimativa de ETo.

3. Em dias de céu nublado e aberto, os métodos de Camargo e da radiação solar são mais eficientes na estimativa da ETo.

4. Métodos desenvolvidos para aplicação em agrupamentos de dados maiores podem ser aplicados em menores intervalos, quando a cobertura de céu se aproxima das condições climáticas locais de origem do método.

\section{Agradescimentos}

Ao Dr. Alaor Moacyr Dall'Antonia Jr., do Instituto Nacional de Meteorologia pela disponibilização dos dados meteorológicos.

\section{Referências}

ALLEN, R.G.; PEREIRA, L.S.; RAES, D.; SMITH, M. Crop evapotranspiration: guidelines for computing crop water requirements. Rome: FAO, 1998. 301p. (FAO. Irrigation and drainage paper, 56).

BARROS, V.R.; SOUZA, A.P.; FONSECA, D.F.; SILVA, L.B.D. Avaliação da evapotranspiração de referência na região de Seropédica, Rio de Janeiro, utilizando lisímetro de pesagem e modelos matemáticos. Revista Brasileira de Ciências Agrárias, v.4, p.198-203, 2009.

CAMARGO, A.P. de; CAMARGO, M.B.P. de. Uma revisão analítica da evapotranspiração potencial. Bragantia, v.59, p.125-137, 2000.

CAMARGO, A.P. de; SENTELHAS, P.C. Avaliação do desempenho de diferentes métodos de estimativa da evapotranspiração potencial no Estado de São Paulo, Brasil. Revista Brasileira de Agrometeorologia, v.5, p.89-97, 1997.

CARVALHO, D.F. de; SILVA, L.D.B. da; FOLEGATTI, M.V.; COSTA, J.R.; CRUZ, F.A. da. Avaliação da evapotranspiração de referência na região de Seropédica-RJ, utilizando lisímetro de pesagem. Revista Brasileira de Agrometeorologia, v.14, p.108-116, 2006.

CARVALHO, D.F. de; SILVA, L.D.B.; GUERRA, J.G.M.; CRUZ, F.A.; SOUZA, A.P. Instalação, calibração e funcionamento de um lisímetro de pesagem. Engenharia Agrícola, v.27, p.363-372, 2007.

CHANG, J. Evapotranspiration. In: CHANG, J. Climate and agriculture: an ecological survey. $2^{\text {nd }}$ ed. Chicago: Aldine Publishing, 1971. p.129-143.

CONCEIÇÃO, M.A.F. Evapotranspiração de referência com base na radiação solar global estimada pelo método de Bristow-Campbell. Engenharia Agrícola, v.30, p.619-626, 2010.

CONCEIÇÃO, M.A.F.; MANDELLI, F. Comparação entre métodos de estimativa da evapotranspiração de referência em Bento Gonçalves, RS. Revista Brasileira de Agrometeorologia, v.13, p.303-307, 2005.

DOORENBOS, J.; PRUITT, W.O. Guidelines for predicting crop water requirements. $2^{\text {nd }}$ ed. Roma: FAO, 1977. 179p. (FAO. Irrigation and drainage paper, 24).

ESCOBEDO, J.F.; GOMES, E.N.; OLIVEIRA, A.P.; SOARES, J. Modeling hourly and daily fractions of UV, PAR and NIR to global solar radiation under various sky conditions at Botucatu, Brazil. Applied Energy, v.86, p.299-309, 2009.

FIETZ, C.R.; FISCH, G.F. Avaliação de modelos de estimativa do saldo de radiação e do método de Priestley-Taylor para a região de Dourados, MS. Revista Brasileira de Engenharia Agrícola e Ambiental, v.13, p.449-453, 2009.

GAVILÁN, P.; LORITE, I.J.; TORNERO, S.; BERENGENA, J. Regional calibration of Hargreaves equation for estimating reference ET in a semiarid environment. Agricultural Water Management, v.81, p.257-281, 2006.

GOMIDE, R.L.; MAENO, P. Requerimento de água pelas culturas. In: ALBUQUERQUE, P.E.P. de; DURÃES, F.O.M. (Ed.). Uso e manejo de irrigação. Brasília: Embrapa Informação Tecnológica, 2008. p.225-253. 
HARGREAVES, G.H.; ALLEN, R.G. History and evaluation of Hargreaves evapotranspiration equation. Journal of Irrigation and Drainage Engineering, v.129, p.53-63, 2003.

JENSEN, M.E.; HAISE, H.R. Estimating evapotranspiration from solar radiation. Journal of the Irrigation and Drainage Division, v.89, p.15-41, 1963.

KASHYAP, P.S.; PANDA, R.K. Evaluation of evapotranspiration estimation methods and development of crop-coefficients for potato crop in a sub-humid region. Agricultural Water Management, v.50, p.9-25, 2001.

LINACRE, E.T. A simple formula for estimating evaporation rates in various climates using temperature data alone. Agricultural Meteorology, v.18, p.409-424, 1977.

MAKKINK, G.F. Ekzameno de la formula de Penman. Netherlands Journal of Agricultural Science, v.5, p.290-305, 1957.

MENDONÇA, E.A.; DANTAS, R.T. Estimativa da evapotranspiração de referência no município de Capim, PB. Revista Brasileira de Engenharia Agrícola e Ambiental, v.14, p.196-202, 2010.

OLIVEIRA, L.F.C. de; CARVALHO, D.F. de; ROMÃO, P. de A.; CORTÊS, F.C. Estudo comparativo de modelos de estimativa da evapotranspiração para algumas localidades no Estado de Goiás e Distrito Federal. Pesquisa Agropecuária Tropical, v.31, p.121-126, 2001.

PEREIRA, A.R.; VILLA NOVA, N.A.; SEDIYAMA, G.C. Evapotranspiração. Piracicaba: Fealq, 1997. 183p.
PRIESTLEY, C.H.B.; TAYLOR, R.J. On the assessment of surface heat flux and evaporation using large-scale parameters. Monthly Weather Review, v.100, p.81-92, 1972.

SAMANI, Z. Estimating solar radiation and evapotranspiration using minimum climatological data. Journal of Irrigation and Drainage Engineering, v.126, p.265-267, 2000.

STEDUTO, P.; TODOROVIC, M.; CALIANDRO, A.; RUBINO, P. Daily reference evapotranspiration estimates by the Penman-Monteith equation in Southern Italy: constant vs. variable canopy resistance. Theoretical and Applied Climatology, v.74, p. $217-225,2003$

STONE, R.J. Improved statistical procedure for the evaluation of solar radiation estimation models. Solar Energy, v.51, p.289-291, 1993.

TRAJKOVIC, S.; KOLAKOVIC, S. Evaluation of reference evapotranspiration equations under humid conditions. Water Resource Management, v.23, p.3057-3067, 2009.

TURC, L. Estimation of irrigation water requirements, potential evapotranspiration: a simple climatic formula evolved up to date. Annals of Agronomy, v.12, p.13-49, 1961.

VESCOVE, H.V.; TURCO, J.E.P. Comparação de três métodos de estimativa da evapotranspiração de referência para a região de Araraquara - SP. Engenharia Agrícola, v.25, p.713-721, 2005.

ZANETTI, S.S.; SOUSA, E.F.; CARVALHO, D.F. de; BERNARDO, S. Estimação da evapotranspiração de referência no Estado do Rio de Janeiro usando redes neurais artificiais. Revista Brasileira de Engenharia Agrícola e Ambiental, v.12, p.174-180, 2008.

Recebido em 22 de setembro de 2010 e aprovado em 31 de janeiro de 2011 